\title{
Simulations of a Miniaturized Cylindrical Hall Thruster
}

\author{
Laurent Garrigues, Gerjan J. M. Hagelaar, Jean Pierre Boeuf, Yevgeny Raitses, \\ Artem Smirnov, and Nathaniel J. Fisch
}

\begin{abstract}
A miniaturized cylindrical Hall thruster (CHT) is studied using a 2-D hybrid model. The simulation results are compared with experimental results for 100-W laboratory CHT. The channel of this thruster has a short coaxial part and a longer cylindrical region with a larger volume-to-surface ratio to reduce erosion. The 2-D model combines a fluid description of the electrons (continuity and momentum equations) and a particle description of the ions and the neutral atoms. The simulations confirm that the ionization takes place in the annular part of the channel, while the unusually high propellant utilization efficiency is found to be due to doubly charged ions.
\end{abstract}

Index Terms-Cylindrical Hall thruster (CHT), simulations.

\section{NOMENCLATURE}

$B$

$e$

$I_{\text {back }}$ and $I_{\text {front }}$

$I_{d}$ and $I_{i}$

$k_{B}$

$m_{a}$

$m_{e}$ and $M$

$n, n_{0}, n_{e}$, and $n_{i}$

$N$

$S$

$T$

$T_{e}$

$U$

$V$

$V_{d}$

$W$

$x$ and $r$

$\mathrm{Xe}^{+}$and $\mathrm{Xe}^{2+}$

$\alpha_{\varepsilon}$

$\beta$

Manuscript received November 12, 2007; revised January 25, 2008 and July 8, 2008. First published October 21, 2008; current version published November 14, 2008.

L. Garrigues, G. J. M. Hagelaar, and J. P. Boeuf are with the LAboratoire PLAsma et Conversion d'Energie, Université de Toulouse, CNRS, 31062 Toulouse Cedex 9, France (e-mail: laurent.garrigues@laplace.univ.tlse.fr gerjan.hagelaar@laplace.univ-tlse.fr; jpb@laplace.univ-tlse.fr).

Y. Raitses, A. Smirnov, and N. J Fisch are with the Princeton Plasma Physics Laboratory, Princeton University, Princeton, NJ 08543 USA (e-mail: yraitses@pppl.gov; asmirnov@trialphanenergy.com; fisch@princeton.edu).

Digital Object Identifier 10.1109/TPS.2008.2003976

$\varepsilon$
$\Gamma_{e}$ and $\Gamma_{i}$
$\kappa$
$\bar{\mu}, \mu_{/ /}$, and $\mu_{\perp}$
$\nu_{B}$
$\nu_{m}$

$\eta, \eta_{c}$, and $\eta_{u}$ $\omega_{c}$

$\Omega$

\author{
Electron mean energy. \\ Electron and ion fluxes, respectively. \\ Inelastic effective energy loss coefficient. \\ Electron mobility tensor, parallel electron \\ mobility, and perpendicular electron \\ mobility, respectively. \\ Equivalent Bohm collision frequency. \\ Electron-atom momentum transfer \\ frequency. \\ Anode, current utilization, and propellant \\ utilization efficiencies, respectively. \\ Cyclotron pulsation. \\ Hall parameter.
}

\section{INTRODUCTION}

A SCALE-DOWN of Hall effect thrusters-HETsoperating at a lower electric power is suitable for low-mass spacecraft or multiple microspacecraft flying in constellations for scientific missions [1]. The reduction of electric power implies a decrease in the discharge current. The consequence is that a low neutral mass flow must be injected. To maintain a high propellant utilization efficiency (which means a large ionization of the injected mass flow) in a shorter channel length, the magnetic field must be increased. The magnetic saturation of the circuit near the inner pole induces challenges to reach an optimal magnetic field magnitude. Moreover, because there is no room for an inner coil, a scale-down of the thruster size leads also to some difficulty to achieve a desired magnetic field shape. The magnetic field in the exhaust region cannot be radial, leading to large ion losses and inner wall erosion. Lowpower HETs, based on conventional annular channel, operating in the range of 100-300 $\mathrm{W}$ offer low anode efficiency $\eta$ [2].

Another concept of electromagnetic thruster based on axial electric field and radial magnetic field as in a HET has been proposed a few years ago. This concept named cylindrical Hall thruster (CHT) [3] features a channel with a short annular region and a longer cylindrical region as in Fig. 1. The xenon mass flow is injected through the anode, and the ceramics of the channel are made up of boron nitride as in conventional HETs. Two coils (a front and a back one) are connected to a separate power supply. A cusp magnetic field shape is obtained with counterdirected coil currents, as shown in Fig. 1. Compared to the conventional annular geometry, the CHT offers a larger volume-to-surface ratio and, therefore, potentially smaller ion losses and wall erosion of the inner part [3], [4]. Variants of the CHT with and without a short annular channel were 
(a)

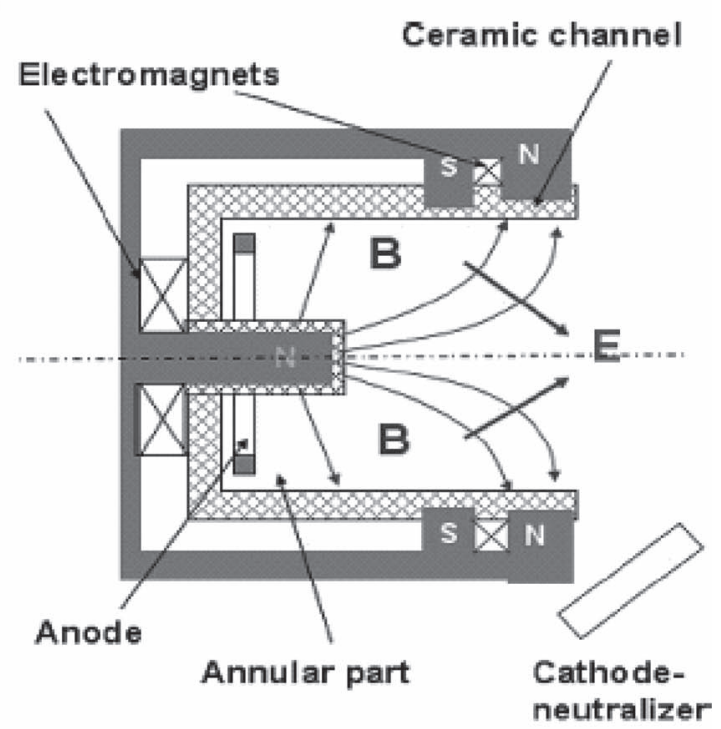

(b)

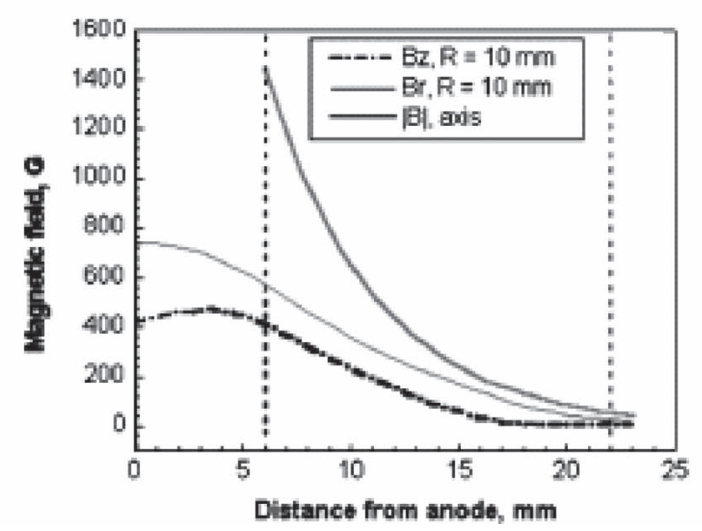

Fig. 1. (a) Schematic of the CHT (from [3]). (b) Magnetic field profiles in the CHT $\left(I_{\text {back }}=2.5 \mathrm{~A}\right.$ and $\left.I_{\text {front }}=-1 \mathrm{~A}\right)$. The dashed lines at $x=6$ and $22 \mathrm{~mm}$ show the edge of the annular channel part and the thruster exit, respectively ([4]).

proposed and developed [3]-[6]. The short annular channel is used to maintain high propellant utilization efficiency in the CHT [3], [4]. On the other hand, the absence of the annular channel may be advantageous with respect to the thruster lifetime [5], [6].

The miniaturized CHT of $2.6-\mathrm{cm}$ diameter (called microCHT in the rest of this paper) was developed and characterized at the Princeton Plasma Physics Laboratory (PPPL). For this micro-CHT, comprehensive measurements of performance, plume characteristics, including ion angular distribution and ion energy distribution function, and plasma properties of the thruster discharge are described elsewhere [4], [7]-[10]. In parallel to the experimental studies, theoretical work has been carried out in order to understand the electron cross-field transport in such complex magnetic field topology [9], [10].

The main conclusions of these experimental and theoretical studies can be summarized as follows.

1) The propellant utilization (defined as the ratio of ion current considering singly charged ions to the propellant mass flow expressed in units of electric current) in the
micro-CHT is higher than in a conventional annular HET of same diameter. Moreover, the propellant utilization in the micro-CHT is higher than one, and a significant fraction of doubly charged ions seems to be present [8], [10].

2) The performance of the micro-CHT is comparable with that of the state-of-the art conventional annular Hall thrusters of similar power levels [4].

3) The plasma density is nonuniform in the radial direction, with the peak being at the thruster axis. The potential drop is concentrated mainly in the cylindrical part of the channel and in the plume. The ion bombardment on the ceramic walls should be reduced in comparison with annular HET [7]. The diamagnetic force is assumed to play an important role in the electron confinement [9].

4) The ion beam divergence, defined as the angle which contains $90 \%$ of the ion current, is large $70^{\circ}-80^{\circ}$ (compared to $45^{\circ}$ for standard HETs) which, of course, leads to a thrust reduction in the axial direction. In the most recent experiments [8], an important 20\%-30\% plume narrowing leading to substantial $50 \%-60 \%$ increase of the thruster anode efficiency at 100-200 W has been observed. These improvements were achieved by increasing the discharge current over and above what is normally required for sustaining the steady-state discharge.

5) Bohm-type anomalous diffusion of the electron transport with a collision frequency $\nu_{B}$ on the order of the Bohm value $\left(\nu_{B} \sim \omega_{c} / 16-\omega_{c}\right.$ is the cyclotron pulsation) matches the measured experimental current [9], [10].

A 2-D model has been developed in the context of conventional HETs [11] and extended to double-stage HET [12]. In this paper, we use the full 2-D model to study the micro-CHT working. This paper is organized as follows. We describe the model in Section II, the results are detailed and commented in Section III, and we finally end this paper with a conclusion in Section IV.

\section{Description OF THE MOdel}

In the 2-D hybrid model, the treatment of the heavy species (singly charged ions $\mathrm{Xe}^{+}$, doubly charged ions $\mathrm{Xe}^{2+}$, and neutrals) is based on particle tracing as done in PIC simulation (see Section II-A). The electron transport is described with fluid equations, and the electric potential profile in two dimensions (without assuming a Boltzmann relation along the magnetic lines) is calculated from a current conservation equation forcing quasi-neutrality (see Section II-B). We end this section (see Section II-C) with the procedure used to fit the anomalous cross-field electron conductivity and the electron-wall energy losses by comparisons between calculations and experimental results.

\section{A. Ion and Neutral Transport}

The motion of heavy particles $\mathrm{Xe}, \mathrm{Xe}^{+}$, and $\mathrm{Xe}^{2+}$ is solved in two dimensions in space and three dimensions in velocity. 
The computational domain assumes a cylindrical symmetry. The domain goes from the thruster centerline to $6.0 \mathrm{~cm}$ in the radial direction $r$ and from the anode to $4.5 \mathrm{~cm}$ in the axial direction $x$. The domain contains the channel of the micro-CHT (annular and cylindrical regions) and the cathode. The cathode is positioned at $r=5.4 \mathrm{~cm}$ and $x=3.5 \mathrm{~cm}$. We typically use 60 cells in the $x$-direction and 80 cells in the $r$-direction. The neutrals are injected in the anode plane, assuming a Maxwellian distribution of the flux in the axial direction for a given temperature equal to $300 \mathrm{~K}$. We also account for the background neutral atoms due to the background pressure of the experimental facility by injecting an additional neutral flux at the thermal velocity (temperature of $300 \mathrm{~K}$ ) from the boundaries of the computational domain. The mass flow of neutral atoms through the cathode with a mass flow of $0.2 \mathrm{mg} / \mathrm{s}$ has also been included in some simulations (see Section III-B). The effect of the neutral atoms flowing through the cathode orifice has been considered assuming that the temperatures of neutral atoms are in equilibrium with the walls (a rough estimation gives a temperature that is approximately $1200 \mathrm{~K}$ [13]). Neutrals colliding with the walls are reflected back in the domain.

The ion source term is calculated assuming a Maxwellian distribution function for the electrons. The origin of $\mathrm{Xe}^{+}$is the ionization of the ground state of xenon, whereas the origin of $\mathrm{Xe}^{2+}$ is the direct double ionization of the ground state of xenon and the stepwise ionization of $\mathrm{Xe}^{+}$. We neglect the effect of triply charged ions. The ionization threshold for the ground state is $70 \mathrm{eV}$ for xenon. Due to the large Larmor radius, the magnetic field effect on the ions is neglected. The ion trajectories are followed until the xenon ions leave the computational domain or impinge the walls. When ions impact on walls, they are neutralized, and new neutral atoms at the wall temperature $(300 \mathrm{~K})$ are reemitted. The angular distribution is assumed semi-isotropic.

\section{B. Electron Transport}

The electron fluid transport is described with the continuity, momentum, and energy equations. The equation for the electron conservation is

$$
\frac{\partial n_{e}}{\partial t}+\nabla \cdot \boldsymbol{\Gamma}_{e}=S
$$

where $n_{e}$ is the electron density, $\Gamma_{e}$ is the electron flux, and $S$ is the ionization source term.

The electron momentum equation is written-in the form of drift-diffusion approximation-as

$$
\Gamma_{e}=\bar{\mu}\left(n_{e} \nabla V-\nabla\left(n_{e} T_{e}\right)\right)
$$

where $T_{e}$ is the electron temperature. We recognize the electric force and the kinetic pressure gradient in the first and second terms of (2), respectively. Due to the magnetic field, the mobility $\bar{\mu}$ is not a scalar but a tensor [14], where the components parallel and perpendicular to the magnetic field are given by the following relations:

$$
\begin{aligned}
& \mu_{/ /}=\frac{e}{m_{e} \nu_{m}} \\
& \mu_{\perp}=\frac{1}{1+\Omega^{2}} \mu_{/ /}=\frac{e \nu_{m} / m_{e}}{\nu_{m}^{2}+\omega_{c}^{2}}
\end{aligned}
$$

where $\Omega$ is the Hall parameter (with $\Omega=\omega_{c} / \nu_{m}-\nu_{m}$ being the electron-neutral momentum collision frequency). For typical conditions, the $\Omega$ parameter varies between $10^{2}$ and $10^{4}$. The perpendicular mobility is therefore lower by orders of magnitude than the parallel one.

Combining (1) and (2) and forcing quasi-neutrality $(n \approx$ $\left.n_{i} \approx n_{e}\right)$, the electric potential $V$ is calculated to solve the following:

$$
\nabla \cdot \boldsymbol{\Gamma}_{e}=\nabla \cdot\left\lfloor\bar{\mu}\left(n \nabla V-\nabla\left(n T_{e}\right)\right)\right\rfloor=\nabla \cdot \boldsymbol{\Gamma}_{i}
$$

In (5), the plasma density $n$ and the ion flux $\Gamma_{i}$ are deduced from the ion transport. The aforementioned elliptic equation is solved on the same computational grid as the grid used to transport ion and neutrals. The difficulty in the resolution of (5) comes from the strong anisotropy of the mobility tensor $\bar{\mu}$. An accurate numerical scheme must be used; otherwise, the crossfield transport (in the perpendicular direction) is determined by the numerical errors, not by the physical cross-field transport [15]. Since we consider a Maxwellian distribution function for the electrons, mirror forces are not included in this approach. A description of the mirror force requires separate equations for the electron energy in the direction parallel and perpendicular to the magnetic field lines.

The boundary conditions are zero potential at the cathode and discharge potential at the anode. On the dielectric walls, on the front plane, and on the free-space edge of the computational domain, the electron flux $\Gamma_{e}$ is set equal to the ion flux $\Gamma_{i}$.

In order to determine the electron temperature $T_{e}$ (or the electron mean energy $\varepsilon=3 / 2 \beta T_{e}$, where $\beta$ is the Boltzmann constant), we solve an energy equation

$$
\begin{aligned}
\frac{\partial(n \varepsilon)}{\partial t}+\frac{5}{3} \nabla \cdot\left(\boldsymbol{\Gamma}_{e} \varepsilon\right)-\frac{10}{9 e} \nabla & \cdot(\mu n \varepsilon \nabla \varepsilon) \\
& =e \boldsymbol{\Gamma}_{e} \cdot \nabla V-N n \kappa-n W
\end{aligned}
$$

where $N$ is the neutral gas density. The last two terms in the energy equation represent energy loss by collisions with gas particles and with the walls, respectively, where $\kappa$ and $W$ are the effective energy loss coefficients. The inelastic rates between electrons and neutrals are calculated assuming a Maxwellian distribution and a given set of cross sections. The inelastic cross sections (single direct ionization and excitation) come from Puech and Mizzi [16]. The double-direct-ionization cross sections from the xenon ground state come from Wetzel et al. [17]. The cross sections for ionization of $\mathrm{Xe}^{+}$are taken from Achenbach et al. [18]. The boundary conditions are the following: We assume a given electron mean energy of $10 \mathrm{eV}$ on the 


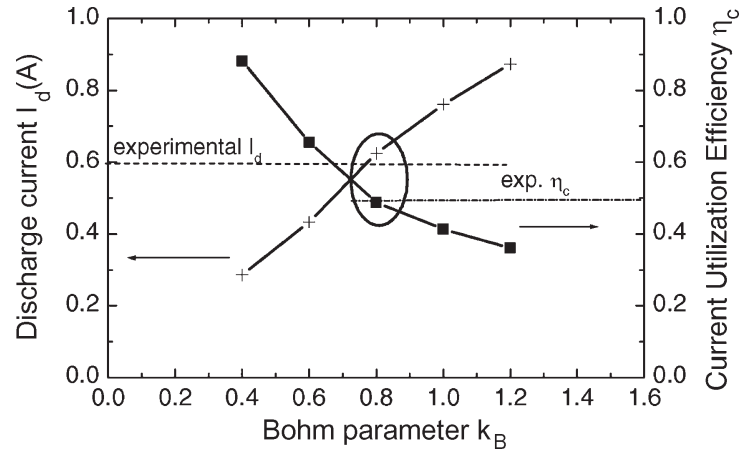

Fig. 2. Calculations of the discharge current $I_{d}$ and current utilization efficiency $\eta_{c}$ for different values of the $k_{B}$ coefficient. The conditions are the following: a discharge voltage $V_{d}$ of $250 \mathrm{~V}$, a mass flow of xenon of $0.4 \mathrm{mg} / \mathrm{s}$, and a background pressure of $9 \mathrm{mPa}$. The experimental values are given in [4].

anode plane, $10 \mathrm{eV}$ in the cathode, and $5 \mathrm{eV}$ in the near plume region. The energy flux to the wall $q_{w}$ is

$$
q_{w}=\frac{5}{3} \boldsymbol{\Gamma}_{e} \cdot \varepsilon
$$

\section{Cross-Field Electron Mobility and Electron-Wall Energy Losses}

As in conventional HETs, the electron cross-field transport is still an open problem in the micro-CHT. A Monte Carlo model of the electron dynamics has been developed in a previous study in order to quantify the mechanism responsible of the electron transport in the direction perpendicular to the magnetic field [9]. The Monte Carlo model accounts for elastic and inelastic collisions with neutrals (assuming a constant neutral density into the computational domain), electron-wall collisions (including secondary electron emission and electron attachment), and Bohm diffusion (through an equivalent collision frequency). The main conclusion of this paper is that the main mechanism which can explain the cross-field transport is the Bohm diffu$\operatorname{sion} \nu_{B} \sim \omega_{c} / 16$, with the electron-wall interactions playing a role only on the electron energy losses.

Based on the same idea, in the hybrid model, we treat the electron cross-field transport with a frequency related to a momentum transfer between electrons and neutrals $\left(\nu_{m}\right.$-the momentum transfer frequency is assumed constant $k_{m}=2.5 \times$ $10^{-13} \mathrm{~m}^{3} \cdot \mathrm{s}^{-1}$ ) and due to field fluctuations (with an equivalent frequency $\nu_{B}=k_{B} \omega_{c} / 16$, where $k_{B}$ is a fitting parameter)

$$
\mu_{\perp}=\frac{e \nu / m_{e}}{\nu^{2}+\omega_{c}^{2}} \approx \frac{m_{e} \nu}{e B^{2}}=\frac{m_{e}\left(\nu_{m}+\nu_{B}\right)}{e B^{2}} .
$$

In order to quantify the $k_{B}$ parameter, we have calculated the discharge current $I_{d}$ and the current utilization efficiency $\eta_{c}$ defined as $I_{i} / I_{d}$ as a function of $k_{B}$. These simulation results are compared with experimental results for the discharge voltage of $250 \mathrm{~V}$ and the anode xenon gas flow rate of $0.4 \mathrm{mg} / \mathrm{s}$ [4], [7], [9], [10]. The simulated values of $I_{d}$ and $\eta_{c}$ give the best agreement with experimental results for $k_{B}=0.8$ (see Fig. 2), confirming a $k_{B}$ parameter that is few times larger than those of conventional HETs [19] $\left(k_{B} \sim 0.1-0.2\right)$. We

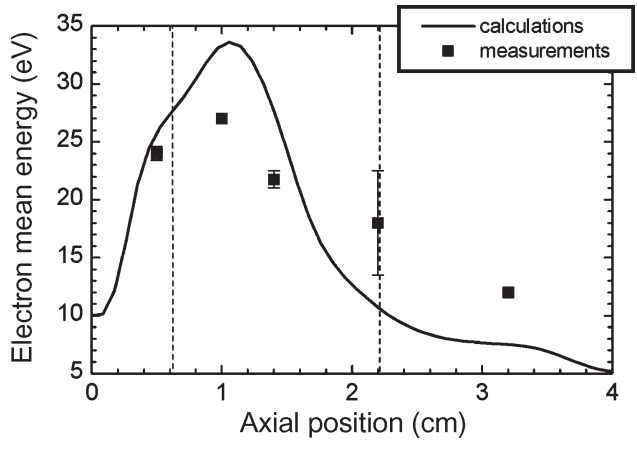

Fig. 3. Calculated and measured [7] profiles of the electron mean energy near the outer wall. The dashed lines at $x=0.6$ and $2.2 \mathrm{~cm}$ represent the edge of the annular channel region and the thruster axis, respectively. The conditions are the same as those in Fig. 2. The vertical bars indicate the uncertainty of the electron mean energy measurements.

remark that the magnetic field reaches $700 \mathrm{G}$ in the annular region, which is three or four times higher than the magnetic field magnitude in the exhaust plane of a conventional HET. The cross-field transport must be increased in the same ratio in order to explain the measured discharge current. We assume that the $k_{B}$ parameter remains constant when the discharge voltage varies (see Section III).

The fluid model shows that energy losses due to electron-atom collisions are not sufficient to reproduce experimental results. To represent energy losses due to electron-wall interactions, we use in this paper the same empirical energy loss coefficient as in [11]. The energy loss per second per electron is taken as

$$
W=\alpha_{\varepsilon} 10^{7} \varepsilon \exp \left(-\frac{U}{\varepsilon}\right)
$$

where $\alpha_{\varepsilon}$ and $U$ are the constant fitting parameters. We have compared the calculated and the measured electron mean energy $\left(\varepsilon=3 / 2 \beta T_{e}\right)$ to fix the $\alpha_{\varepsilon}$ and $U$ parameters. Fig. 3 shows an acceptable agreement between the measured and the calculated profile of $\varepsilon$ for $\alpha_{\varepsilon}=0.7$ and $U=20 \mathrm{eV}$ (the conditions are the same as those in Fig. 2, and $k_{B}=0.8$ ).

\section{RESUlTS AND DISCUSSION}

We performed calculations for a given set of coil currents $I_{\text {back }}=2.5 \mathrm{~A}, I_{\text {front }}=-1 \mathrm{~A}$, for two anode mass flows of 0.4 and $0.6 \mathrm{mg} / \mathrm{s}$; the discharge voltage varies from 200 to $300 \mathrm{~V}$. We present in Section III-A the plasma characteristics for an anode mass flow of $0.4 \mathrm{mg} / \mathrm{s}$ and a discharge voltage $V_{d}$ of $250 \mathrm{~V}$. We also compare simulation results with experimental measurements of electric potential and plasma density. The current-voltage characteristic and the propellant utilization efficiency are presented in Section III-B. We then analyze in the same section the origin of a large propellant utilization efficiency in the micro-CHT.

\section{A. Plasma Characteristics}

The time-averaged spatial distributions of electric potential, ionization source term, and neutral atom and ion densities for 

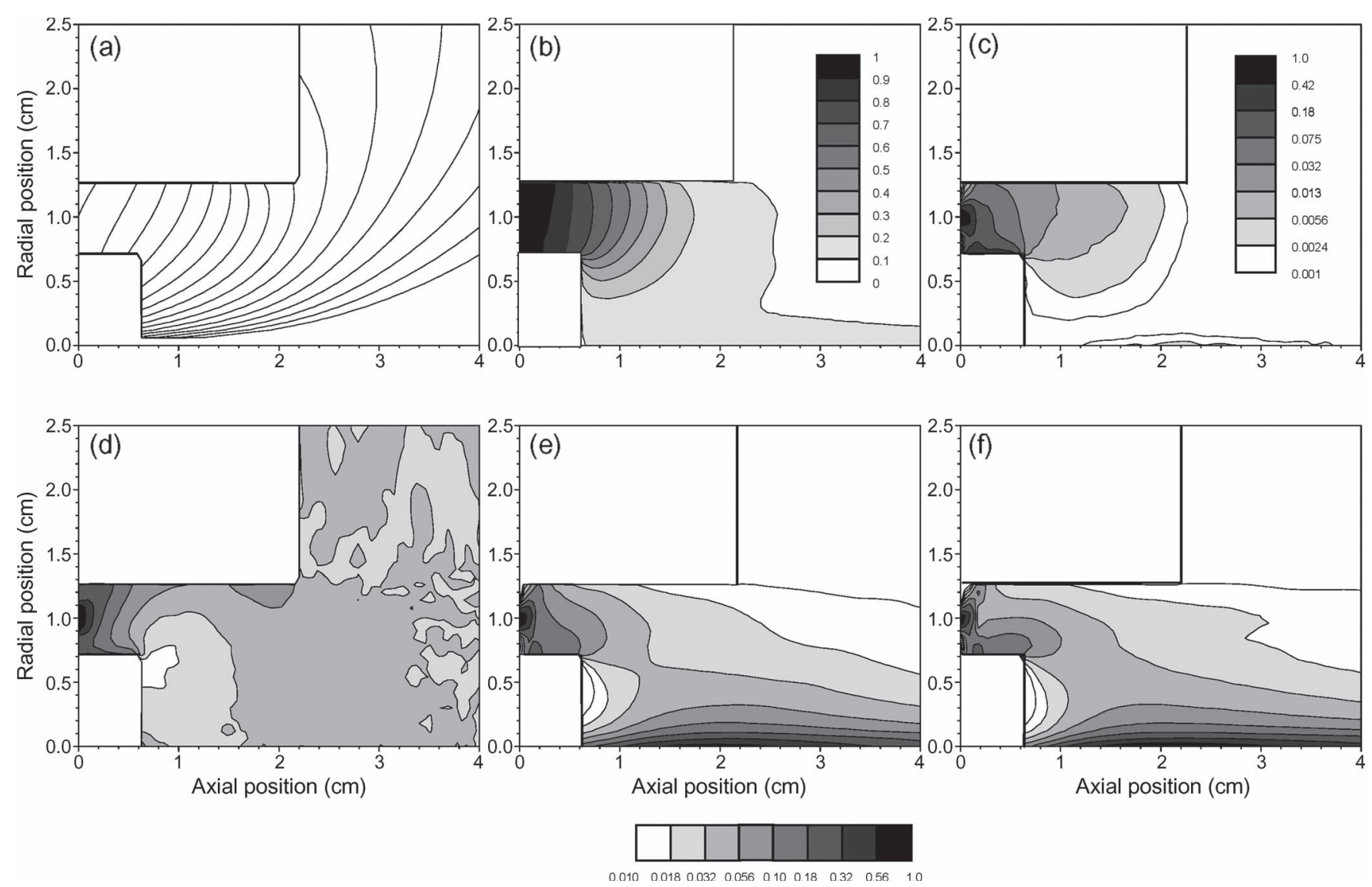

Fig. 4. Plasma characteristics on the subset of the computational domain for a discharge voltage $V_{d}$ of $250 \mathrm{~V}$, for an anode mass flow of xenon of $0.4 \mathrm{mg} / \mathrm{s}$, and for a background pressure of $9 \mathrm{mPa}$.(a) Magnetic field lines. (b) Electric potential (max: $250 \mathrm{~V}$ ). (c) Ionization source term log scale 3 decades (max: $1.6 \times 10^{25} \mathrm{~m}^{-3} \mathrm{~s}^{-1}$ ). (d) Neutral atom density log scale 2 decades (max: $7 \times 10^{19} \mathrm{~m}^{-3}$ ). (e) $X e^{+}$density log scale 2 decades $\left(\right.$ max: $10^{19} \mathrm{~m}-3$ ). (f) $X e^{2+}$ density $\log$ scale 2 decades (max: $6 \times 10^{17} \mathrm{~m}^{-3}$ ).

the interior and near field of the micro-CHT configuration are shown in Fig. 4 for $V_{d}=250 \mathrm{~V}$, an anode xenon mass flow of $0.4 \mathrm{mg} / \mathrm{s}$, and a background pressure of $9 \mathrm{mPa}$. The injection of atoms through the cathode is not included in this section. Fig. 4(a) shows the magnetic field lines. The computational domain is not limited by the magnetic field lines which intercept the cathode, as in previous studies where the electric potential was calculated assuming a Boltzmann relation for the electrons [11], [20], [21]. The electric potential shown in Fig. 4(b) demonstrates that the potential drop is concentrated in the region of high magnetic field which covers the annular and cylindrical parts, with a nonnegligible drop being outside the exit plane. We also see that the electric potential contours do not follow the magnetic field lines inside the coaxial part and outside the micro-CHT channel [compare Fig. 4(a) and (b)]. We observe that the electric potential variation along a magnetic field line which intercepts the magnetic inner pole and the cathode is few tens of volts.

The maximum of ionization is achieved in the annular region, as shown in Fig. 4(c). In this region, the neutral atom density is large [see Fig. 4(d)], the electron energy exceeds $10 \mathrm{eV}$ (as we note in Fig. 3), and the electrons are strongly confined by the strong magnetic field magnitude. The neutral density is maximum in front of the region of atom injection as expected $\left(\sim 10^{20} \mathrm{~m}^{-3}\right)$ and then strongly decreases due to intense ionization. We note a region of nonnegligible atom density due to ion recombination near the outer wall of the cylindrical region. The neutral atom density in the outside region is about $2 \times 10^{18} \mathrm{~m}^{-3}$ arising from the background pressure flow. We observe in Fig. 4(e) two peaks of $\mathrm{Xe}^{+}$ion density - the first one in the annular zone due to the ionization of the neutral gas (with density being in the range of $10^{19} \mathrm{~m}^{-3}$ ) and the second one in the region of the axis of symmetry $\left(\sim 5 \times 10^{18} \mathrm{~m}^{-3}\right)$ due to the convergent ion flux. Although the xenon mass flowing through the anode is relatively low (ten times less than the mass flow injected for 1-kW HETs), the $\mathrm{Xe}^{+}$ion density is high due to the small channel cross section and volume of the annular region of the micro-CHT $\left(2 \mathrm{~cm}^{-3}\right.$ compared with $50 \mathrm{~cm}^{-3}$ of the $9-\mathrm{cm}-$ diameter CHT). A possible effect of $\mathrm{Xe}^{2+}$ has been invoked to a possible explanation of the high propellant utilization efficiency of the micro-CHT [4], [10]. We have shown in Fig. 4(f) the profile of $\mathrm{Xe}^{2+}$ ion density. We observe that the profile has the same shape with the $\mathrm{Xe}^{+}$ion density profile, which indicates that the $\mathrm{Xe}^{2+}$ ions are mainly produced by ionization of the singly charged ions. The electron energy is not large enough to produce a substantial fraction of $\mathrm{Xe}^{2+}$ ions by direct impact ionization of the xenon atom. The interesting result is that the fraction of doubly charged ions already represents a few percent of the total ion density for an anode mass flow of xenon of $0.4 \mathrm{mg} / \mathrm{s}$. Same calculations for a mass flow of $0.6 \mathrm{mg} / \mathrm{s}$ lead to a higher density in the coaxial part with a percentage of doubly charged ions which attains $10 \%$ of the total ion density. 

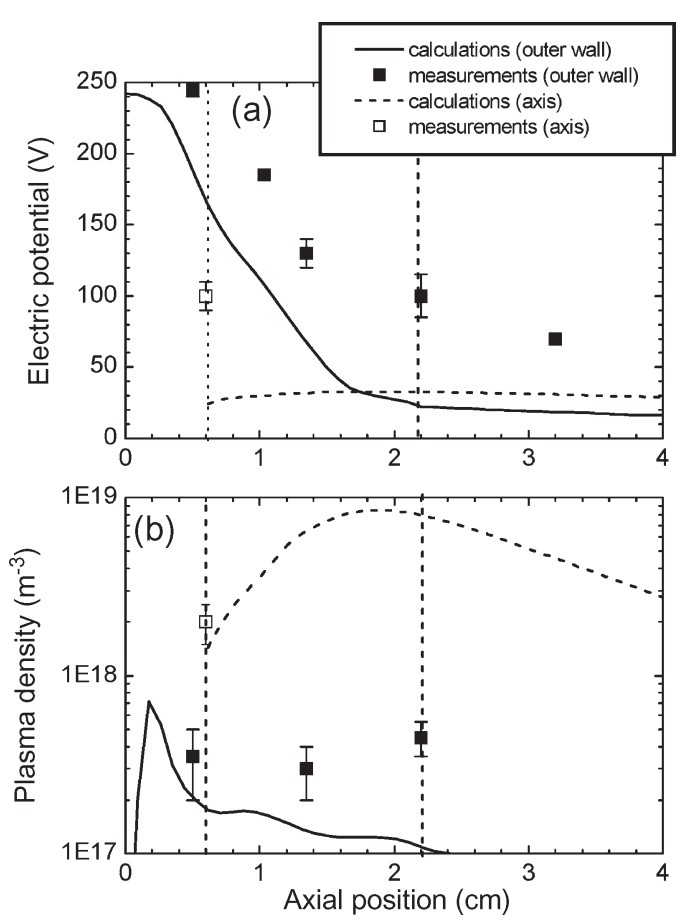

Fig. 5. Comparisons between the calculated and measured profiles [7] of (a) electric potential and (b) plasma density for a discharge voltage $V_{d}$ of $250 \mathrm{~V}$, a mass flow of xenon of $0.4 \mathrm{mg} / \mathrm{s}$, and a background pressure of $9 \mathrm{mPa}$. The vertical bars indicate the uncertainty of the measurements.

There are considerable discrepancies between simulated and measured [7] results of the electric potential distribution [Fig. 5(a)]. In the simulations, the voltage potential drop in the annular channel is much larger than in the experiments. In addition, the electric potential at the channel exit is almost four times lower than that measured for the $2.6-\mathrm{cm}$ micro-CHT. We can make two remarks on the possible causes of these discrepancies. The first remark is related to the effect of anomalous transport coefficient on the electric potential profile, as it has been shown in [19] and [21]. We have taken the same anomalous Bohm coefficient in the annular and cylindrical regions, but there is no evidence to do so. A higher Bohm coefficient inside the annular region, representing a larger electron conductivity near the anode, would lead to a smaller potential drop inside this region. The second remark concerns the pressure force (2). We underestimate the electron energy in the region near the exhaust plane (see Fig. 3) by a factor of two, which can maybe explain some differences between the simulated and measured values of electric potential in this region. Again, we use the same parameter to account for the electron energy losses due to electron-wall interactions inside and outside the coaxial part. The boundary condition on the electron energy in the plume may also affect the simulation results.

As observed experimentally, the calculated plasma density near the outer wall is lower than on the thruster axis by more than one order of magnitude [see Fig. 5(b)]. We note some difference beyond the exit plane. The measured plasma density reaches $6 \times 10^{17} \mathrm{~m}^{-3}$, which is five times the calculated plasma density at the same location. It is maybe due to the ionization of neutrals coming from ion recombination. We observe a non-
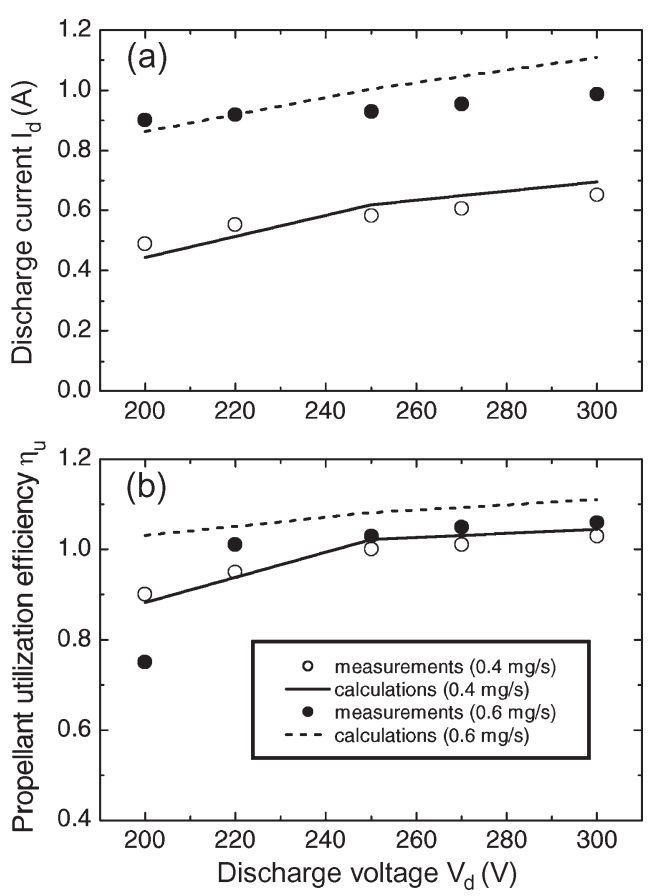

Fig. 6. (a) Current-voltage characteristic for two anode mass flows of xenon. (b) Propellant utilization efficiency versus discharge voltage. The background pressure is $9 \mathrm{mPa}$. The propellant utilization efficiency does not include the cathode gas flow rate.

negligible neutral density in the cylindrical region near the outer wall [see Fig. 4(d)], but it seems that the ionization of the neutral atoms is still low due to a low electron energy in the channel exit region. Despite the fact that we do not account for the diamagnetic force effect, we do think that the main origin of the discrepancies between the experiments and the calculations are related to the bad estimation of the parameters involved in the fluid description of the electrons ( $k_{B}$ and $\alpha_{\varepsilon}$ in the momentum and energy equations, respectively).

\section{B. Ion Current and Current-Voltage Characteristics}

We have shown in Fig. 6(a) the discharge current as a function of the discharge voltage for two anode mass flows of xenon $(0.4$ and $0.6 \mathrm{mg} / \mathrm{s})$ and for discharge voltages in the range of 200-300 V. The agreement between the measured and calculated currents is reasonable, given that the coil currents are kept constant in all calculations.

A more interesting result is shown in Fig. 6(b), where we have calculated the propellant utilization efficiency $\eta_{u}$ in the same conditions as in Fig. 6(a), assuming singly charged ions. We recall that $\eta_{u}$ is defined as

$$
\eta_{u}=\frac{I_{i} M}{e m_{a}}
$$

where $I_{i}$ is the total ion current, $M$ is the mass of the propellant gas, and $m_{a}$ is the anode mass flow of xenon. We observe in Fig. 6(b) that $\eta_{u}$ is higher than one at high voltage. The possible mechanisms responsible for a large $\eta_{u}$ are the presence of doubly charged ions and/or a residual neutral atom mass flow which can result from the neutral background pressure and from the neutral flowing through the cathode. We then have examined 
TABLE I

Calculations of Propellant Utilization EfFiciency $\eta_{u}$ For Different Conditions, a Discharge Voltage $V_{d}$ of 250 V, A MASS Flow OF XENON OF $0.6 \mathrm{mg} / \mathrm{s}$

\begin{tabular}{|c|ccc|c|}
\hline CASE & $\begin{array}{c}\text { Facility background } \\
\text { pressure }(\mathrm{mPa})\end{array}$ & $\begin{array}{c}\text { xenon cathode } \\
\text { flow }(\mathrm{mg} / \mathrm{s})\end{array}$ & $\begin{array}{c}\text { doubly charged } \\
\text { ions }\end{array}$ & $\begin{array}{c}\text { propellant utilization } \\
\text { efficiency } \eta_{u}\end{array}$ \\
\hline 1 & 0 & 0 & No & 0.95 \\
\hline 2 & 0 & 0.2 & No & 0.95 \\
\hline 3 & 9 & 0 & No & 0.97 \\
\hline 4 & 20 & 0 & No & 1.01 \\
\hline 5 & 30 & 0 & No & 1.19 \\
\hline 6 & 0 & 0 & Yes & 1.05 \\
\hline
\end{tabular}

different scenarios in order to understand the origin of the high propellant utilization efficiency in the micro-CHT.

All the simulations in this section fixed the anode mass flow rate to $0.6 \mathrm{mg} / \mathrm{s}$ and the discharge voltage to $250 \mathrm{~V}$. A summary of the conditions studied and the calculated propellant utilization efficiency is presented in Table I. Note that, in the simulations, for the sake of simplicity, the angle between the thruster and the cathode is $90^{\circ}$, while in the experiments, this angle is actually $30^{\circ}$. As has been recently studied experimentally ([22]), CASE 1 and CASE 3 confirm that the neutral flow from the cathode does not significantly affect the propellant utilization efficiency. In the experiments, the micro-CHT thruster was characterized at high $\left(9 \mathrm{mPa} \sim 7 \times 10^{-5}\right.$ torr $)$ [23] and low (0.4-0.8 $\mathrm{mPa} \sim 3-6 \times 10^{-6}$ torr) [8], [10], [22] background pressure environments. CASE 3, CASE 4, and CASE 5 represent a similar sensibility study of the background pressure on $\eta_{u}$. We note that, for the high background pressure, the propellant utilization efficiency remains below the calculated values shown in Fig. 6(b) $\left(\eta_{u}=1.05\right)$. The effect of the background pressure is determined by the pressure balance in the exit. To reach $\eta_{u}=1.05$, the background pressure must be in the range of $25 \mathrm{mPa}$, which is on the order of one order of magnitude higher than the experimental study conditions. Finally, in CASE 6, we have introduced $\mathrm{Xe}^{2+}$ ions, where we have not observed a large increase in the propellant utilization efficiency. From this study, we can conclude that the $\mathrm{Xe}^{2+}$ ions seem to play a larger role on the observed high propellant utilization efficiency in the micro-CHT than the neutrals coming from the background pressure or external cathode.

\section{CONCLUSiON}

A linear scale-down of Hall thrusters to low power levels requires that the magnitude of the magnetic field is increased as the dimension of the HETs is reduced in order to preserve a high degree of ionization of the neutral flux. This scaling is questionable because there is no room to implement a sophisticated magnetic circuit with inner coils and magnetic screens, which are typically used for larger high-performance Hall thrusters of medium and high power levels. The optimization of the magnetic field configuration is made difficult leading to large ion wall losses and possible erosion of the thruster channel. The anode efficiency $\eta$ of conventional HETs remains low. The micro-CHT was designed to improve efficiency by changing the physical geometry as well to promote higher propellant efficiency.

The aim of this paper is to use a 2-D hybrid model in order to answer some questions or confirm some hypotheses concerning thruster operation. We can summarize the main results as follows.

1) Plasma instabilities seem to play a big role in the special magnetic field configuration of the micro-CHT. In order to reproduce the measured discharge current, a supplementary equivalent collision frequency on the order of $\nu_{B} \sim \omega_{c} / 16$ (Bohm formula) must be taken into account in the fluid description of the electron cross-field transport.

2) The potential drop begins in the annular region and sharply decreases in the cylindrical part. The electric potential in the plume is few tens of electron volts. We observe two peaks of plasma density - the first one in the annular region and the second one on the thruster axis due to the convergent ion flux. A comparison between the experimental and calculated profiles of electric potential and plasma density shows noticeable differences.

3) The calculations show a high level of propellant utilization efficiency. Different scenarios have been proposed to understand the origin of high propellant utilization efficiency. We have seen that the supplementary xenon cathode flow or background pressure does not play a crucial role. The doubly charged ions produced by ionization of $\mathrm{Xe}^{+}$highly influence the level of propellant utilization efficiency.

\section{ACKNOWLEDGMENT}

The 2-D model development has been performed in the frame of the Groupement De Recherche CNRS/CNES/SNECMA/ Universités No. 3161 "Propulsion Spatiale à Plasma." 


\section{REFERENCES}

[1] V. Khayms and M. Martinez-Sanchez, "Fifty-watt Hall thruster for microsatellites," in Micropropulsion for Small Spacecraft, vol. 147, M. Micci and D. Ketsdever, Eds. Washington, DC: AIAA, 2000, ch. 9, pp. 233-254.

[2] D. Jacobson and R. Jankovsky, "Test results of a $200 \mathrm{~W}$ class Hall thruster," presented at the 34th Joint Propulsion Conf. and Exhibit, Cleveland, OH, 1999, Paper AIAA-98-3792.

[3] Y. Raitses and N. J. Fisch, "Parametric investigation of a nonconventional Hall thruster," Phys. Plasmas, vol. 8, no. 5, pp. 2579-2586, May 2001.

[4] A. Smirnov, Y. Raitses, and N. J. Fisch, "Parametric investigation of miniaturized cylindrical and annular Hall thrusters," J. Appl. Phys., vol. 92, no. 6, pp. 5673-5679, Nov. 2002.

[5] Y. Raitses, N. J. Fisch, K. M. Ertmer, and C. A. Burlingame, "A study of cylindrical Hall thruster for low power space applications," presented at the 36th Joint Propulsion Conf. and Exhibit, Huntsville, AL, 2000, Paper AIAA-2000-3421.

[6] A. Shirasaki and H. Tahara, "Operational characteristics and plasma measurements in cylindrical Hall thrusters," J. Appl. Phys., vol. 101, no. 7, p. 073 307, Apr. 2007.

[7] A. Smirnov, Y. Raitses, and N. J. Fisch, "Plasma measurements in a $100 \mathrm{~W}$ cylindrical Hall thruster," J. Appl. Phys., vol. 95, no. 5, pp. 2283-2292, Mar. 2004.

[8] Y. Raitses, A. Smirnov, and N. J. Fisch, "Enhanced performance of cylindrical Hall thrusters," Appl. Phys. Lett., vol. 90, no. 5, p. 221502 , May 2007.

[9] A. Smirnov, Y. Raitses, and N. J. Fisch, "Electron cross-field transport in a low power cylindrical Hall thruster," Phys. Plasmas, vol. 11, no. 11, pp. 4922-4933, Nov. 2004.

[10] A. Smirnov, Y. Raitses, and N. J. Fisch, "Experimental and theoretical studies of cylindrical Hall thrusters," Phys. Plasmas, vol. 14, p. 057 106, May 2007.

[11] G. J. M. Hagelaar, J. Bareilles, L. Garrigues, and J. P. Boeuf, "Two-dimensional model of a stationary plasma thruster," J. Appl. Phys., vol. 91, no. 9, pp. 5592-5598, May 2002.

[12] J. Perez-Luna, G. J. M. Hagelaar, L. Garrigues, and J. P. Boeuf, "Model analysis of a double-stage Hall effect thruster with double-peaked magnetic field and intermediate electrode," Phys. Plasmas, vol. 14, p. 113 502, Nov. 2007.

[13] M. Domonkos, "A particle and energy balance model of the orificed hollow cathode," presented at the 38th Joint Propulsion Conf. and Exhibit, Indianapolis, IN, 2002, Paper AIAA-2002-4240.

[14] M. A. Lieberman and A. J. Lichtenberg, Principles of Plasma Discharges and Materials Processing, 2nd ed. Hoboken, NJ: Wiley, 1994, ch. 5.

[15] G. J. M. Hagelaar, "Modelling electron transport in magnetized lowtemperature discharge plasmas," Plasma Sources Sci. Technol., vol. 16, no. 1, pp. S57-S66, Feb. 2007.

[16] V. Puech and S. Mizzi, "Collision cross sections and transport parameters in neon and xenon," J. Phys. D, Appl. Phys., vol. 24, no. 11, pp. 19741985, Nov. 1991.

[17] R. C. Wetzel, F. Baiocchi, T. R. Hayes, and R. S. Freund, "Absolute cross sections for electron-impact ionization of the rare-gas atoms by the fast-neutral-beam method," Phys. Rev. A, Gen. Phys., vol. 35, no. 2, pp. 559-577, Jan. 1987.

[18] C. Achenbach, A. Muller, E. Salzborn, and R. Becker, "Single ionization of multiply charged xenon ions by electron impact," J. Phys. B, At. Mol. Opt. Phys., vol. 17, no. 7, pp. 1405-1425, Apr. 1984.

[19] C. Boniface, L. Garrigues, G. J. M. Hagelaar, J. P. Boeuf, D. Gawron, and S. Mazouffre, "Anomalous cross field electron transport in a Hall effect thruster," Appl. Phys. Lett., vol. 89, no. 16, p. 161 503, Oct. 2006.

[20] L. Garrigues, G. J. M. Hagelaar, J. Bareilles, C. Boniface, and J. P. Boeuf, "Model study of the influence of the magnetic field configuration and a performance and lifetime of a Hall thruster," Phys. Plasmas, vol. 10, no. 12 , pp. 4886-4892, Dec. 2003.

[21] J. Bareilles, G. J. M. Hagelaar, L. Garrigues, C. Boniface, and P. Gascon, "Critical assessment of a two-dimensional hybrid Hall thruster model: Comparisons with experiments," Phys. Plasmas, vol. 11, no. 6, pp. 30353046, Jun. 2004.

[22] Y. Raitses, A. Smirnov, E. Granstedt, and N. J. Fisch, "Overrun discharge current operation of low power cylindrical Hall thrusters," presented at the 30th International Electric Propulsion Conf., Florence, Italy, 2007, Paper IEPC 007-222.

[23] A. Smirnov, Y. Raitses, and N. J. Fisch, "Enhanced ionization in the cylindrical Hall thruster," J. Appl. Phys., vol. 94, no. 2, pp. 852-857, Jul. 2003.
Laurent Garrigues received the Ph.D. degree from the Université Paul Sabatier, Toulouse, France, in 1998.

He has been with the Centre de Physique des Plasmas et Applications de Toulouse [CPAT; currently the LAboratoire PLAsma et Conversion d'Energie (LAPLACE), Université de Toulouse], Toulouse, France. He was with the Department of Aerospace Engineering, University of Michigan, Ann Arbor, as a Postdoctoral Research Scientist in 2000. He joined the National Center for Scientific Research (CNRS) as a Chargé de Recherche at the CPAT in 2002. His current research interest includes the modeling of nonequilibrium plasmas for applications such as ion sources (satellite propulsion and negative-ion source for ITER), plasma processing (microwave plasma reactors), and vacuum circuit breaker.

Gerjan J. M. Hagelaar received the Ph.D. degree from the Technische Universiteit Eindhoven, Eindhoven, The Netherlands, in 2000.

In 2001, he joined the Centre de Physique des Plasmas et Applications de Toulouse, Toulouse, France. In 2003, he joined the Laboratoire d'Ingénierie des Matériaux et des Hautes Pressions, Villetaneuse, France. In 2004, he joined the National Center for Scientific Research (CNRS), Centre de Physique des Plasmas et Applications de Toulouse (now the LAboratoire PLAsma et Conversion d'Energie (LAPLACE), Université de Toulouse, CNRS, Toulouse). His previous research interests included the modeling of microdischarges for display technology, plasma propulsion, and microwave plasma. His current research interests include the development of the physical and numerical models in nonequilibrium plasmas for numerous applications.

Jean Pierre Boeuf received the degree from the Ecole Supérieure d'Electricité, Gif sur Yvette, France, in 1977, and the Ph.D. degree and the Docteur ès Sciences degree in plasma physics from the Université de Paris XI, Orsay, France, in 1981 and 1985, respectively.

In 1983, he joined the National Center for Scientific Research (CNRS), Laboratoire de Physique des Décharges, Ecole Supérieure d'Electricité, Gif sur Yvette. In 1986, he joined the Centre de Physique des Plasmas et Applications de Toulouse (CPAT), Université Paul Sabatier, Toulouse, France. $\mathrm{He}$ is currently the Directeur de Recherche with the LAboratoire PLAsma et Conversion d'Energie (LAPLACE), Université de Toulouse, CNRS, Toulouse, which is a joint laboratory of the CNRS, Université Paul Sabatier, and Institut National Polytechnique de Toulouse. He is currently in charge of the Research Group in Energetic and Non-equilibrium Plasmas (GREPHE). His current research interests include plasma thrusters for satellite propulsion, plasmas for aerodynamic applications, negative ion source for the ITER neutral beam injection, microdischarges and applications, in collaboration with academic and industrial partners.

Yevgeny Raitses received the Ph.D. degree from the Technion-Israel Institute of Technology, Haifa, Israel.

He has been with the Princeton Plasma Physics Laboratory, Princeton University, Princeton, NJ, since 1998, where he is currently a Research Physicist and the Lead Scientist on the plasma thruster experiment. Previously, he held research positions with the Propulsion Physics Laboratory, Soreq NRC, Yavne, Israel. His current research interests include plasma-wall interactions in gas discharges, physics of Hall thrusters, and plasma diagnostics. 


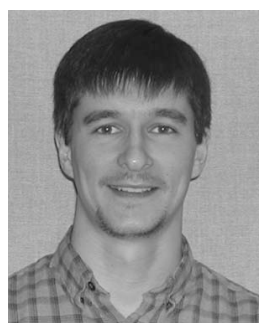

Artem Smirnov received the M.S. degree in physics from the University of Nizhny Novgorod, Nizhny Novgorod, Russia, in 2000 and the Ph.D. degree in plasma physics from Princeton University, Princeton, NJ, in 2006

$\mathrm{He}$ is currently with Tri Alpha Energy, Inc., El Toro, CA. His research interests include lowpressure gas discharge, ion and plasma beam generation, plasma propulsion, and innovative plasma confinement concepts.
Nathaniel J. Fisch received the B.S., M.S., and Ph.D. degrees in electrical engineering and computer science from the Massachusetts Institute of Technology, Cambridge, where he was an MIT National Scholar, in 1972, 1975, and 1978, respectively.

He is a Professor of astrophysical sciences and the Director of the Program in Plasma Physics with Princeton University, Princeton, NJ. He also is the Associate Director for Academic Affairs and the Head of the Hall Thruster Laboratory, Princeton Plasma Physics Laboratory. At Princeton University, he is also an Associated Faculty with the Department of Mechanical and Aerospace Engineering. He is best known for predicting new ways to drive electric current in hot magnetized plasma by means of electromagnetic waves. In addition to plasma thrusters and related plasma devices, his current research interests include plasma-based methods of generating extreme laser intensities and fusion concepts employing magnetically or inertially confined plasma.

Prof. Fisch received the 2005 James Clerk Maxwell Prize of the American Physical Society, the Ernest Orlando Lawrence Award in 2004, the Department of Energy Bronze Medal for Outstanding Mentor in 2002, and the American Physical Society Award for Excellence in Plasma Physics in 1992. A Fellow of the American Physical Society and a Fellow of the NASA Institute for Advanced Concepts, he received a Guggenheim Fellowship in 1985. In 1998, he was the Chair of the Division of Plasma Physics, American Physical Society. 